\title{
Evolução da positividade sorológica para a doença de Chagas numa comunidade rural brasileira
}

\author{
Afonso Dinis Costa Passos, ${ }^{1}$ Jarbas Leite Nogueira, ${ }^{1}$ \\ José Fernando de Castro Figueiredo, ${ }^{2}$ Uilho Antônio Gomes ${ }^{1} e$ \\ Amaury Lelis Dal-Fabbro ${ }^{1}$
}

RESUMO Com o objetivo de avaliar a evolução da positividade sorológica para doença de Chagas na comunidade rural de Cássia dos Coqueiros, Estado de São Paulo, Brasil, foram comparados dois estudos transversais realizados na localidade nos anos de 1971-1972 e 1989-1991. O primeiro levantamento utilizou reação de fixação de complemento para determinar a positividade para sorologia da doença. A prevalência total da doença de Chagas encontrada por este estudo foi de 16,6\%, com variação de 2,9 a 61,9\% (nos grupos etários de 10 a 19 e de 50 a 59 anos de idade, respectivamente). No estudo de 1989-1991, que utilizou imunofluorescência indireta, a prevalência total caiu para 10,1\%, com valor mínimo de 0,4\% (de 10 a 19 anos) e máximo de 44,8\% (acima de 60 anos de idade). Entre pessoas nascidas em Cássia dos Coqueiros, o estudo de 1989-1991 mostrou positividade a partir da idade de 38 anos, com uma única exceção (um homem de 24 anos de idade). A queda no índice de positividade para doença de Chagas em Cássia dos Coqueiros no período analisado pode ser parcialmente devida a fatores sócio-econômicos, como melhoria das condições de vida e progressivo esvaziamento das áreas rurais. Porém, os resultados apontam para a aplicação domiciliar de inseticidas de ação residual, implementada pela primeira vez em 1950, como o fator determinante no combate aos triatomíneos e no controle da transmissão da doença. Os resultados sugerem que a interrupção da transmissão da doença pode ter ocorrido já ao final de 1954, consolidando-se posteriormente através da melhoria das condições sócio-econômicas e da eliminação do Triatoma infestans da área de estudo.

A doença de Chagas, transmitida pelo Triatoma infestans, ocorre no Brasil em uma área de cerca de três milhões de quilômetros quadrados e afeta

\footnotetext{
1 Departamento de Medicina Social, Faculdade de Medicina de Ribeirão Preto, Universidade de São Paulo, Avenida Bandeirantes, 3900, CEP 14049900, Ribeirão Preto, SP, Brasil. Correspondência e pedidos de separatas devem ser enviados para Afonso Dinis Costa Passos neste endereço.

2 Departamento de Clínica Médica, Faculdade de Medicina de Ribeirão Preto, Universidade de São Paulo, Ribeirão Preto, SP, Brasil.
}

aproximadamente cinco milhões de pessoas. Esta doença, decorrente, na sua essência, das péssimas condições sociais a que estão submetidas extensas parcelas da população rural brasileira, é uma das mais importantes endemias encontradas em território brasileiro $(1,2)$.

A região nordeste do Estado de São Paulo apresentava, nas décadas de 40 e 50 , elevados níveis de transmissão da moléstia (3-5). Nessa região, a localidade de Cássia dos Coqueiros, distante cerca de 80 km de Ribeirão Preto, destacava-se como um dos mais importantes focos da endemia, com valores extremamente altos de infestação domiciliar por triatomíneos (as referências da época mencionam infestações prediais da ordem de até 30\%) (3).

Os primeiros estudos sobre a doença de Chagas na região foram iniciados em meados da década de 40. Em 1950, 1952 e 1954, foram realizadas as primeiras borrifações domiciliares com inseticidas de ação residual, seguidas, 
posteriormente, por trabalhos sistematizados de aplicação de organoclorados (4). A aplicação sistematizada de organoclorados se estendeu até 1963.

Todos os fatores descritos acima tiveram influência sobre a transmissão e ocorrência da doença de Chagas na região em questão. O presente trabalho teve como objetivo analisar a evolução da positividade sorológica para doença de Chagas em Cássia dos Coqueiros no decorrer de um período de 20 anos, mediante a comparação dos resultados de dois inquéritos realizados naquela localidade, o primeiro em 1971-1972 e o segundo em 1989$1991(6,7)$.

\section{MATERIAIS E MÉTODOS}

Foram comparados os resultados de dois estudos transversais realizados em Cássia dos Coqueiros, em 19711972 e 1989-1991. Em ambos os estudos, a única limitação para inclusão de participantes foi idade abaixo de 1 ano, devido aos problemas relacionados à coleta de sangue de crianças muito pequenas em condições de trabalho de campo.

O primeiro estudo teve como objetivo básico estudar a prevalência de cardiopatias no Município. A amostra populacional deste estudo, escolhida de maneira casual e probabilística, foi de 823 indivíduos (23,4\% da população total na época do estudo). Estas pessoas foram submetidas a exame físico detalhado (conforme os princípios básicos da semiotécnica, compreendendo inspeção, palpação, percussão e ausculta de todos os segmentos corpóreos aos quais estes processos se aplicam) e à sorologia para doença de Chagas através da reação de fixação do complemento (8), realizada em tubos com antígeno tríplice, de acordo com Almeida, Freitas e Brandão (9).

O segundo inquérito, realizado em 1989-1991, examinou 1951 moradores (69,7\% do total da população do Município na época do estudo) com idade superior a 1 ano. O objetivo deste estudo foi determinar a prevalência de marcadores sorológicos de hepatite $\mathrm{B}$ e estudar possíveis fatores de risco para

TABELA 1. Prevalência de sorologia positiva para a doença de Chagas segundo a idade dos examinados e o período do estudo, Cássia dos Coqueiros, SP, Brasil

\begin{tabular}{|c|c|c|c|c|}
\hline \multirow[b]{3}{*}{ Idade } & \multicolumn{4}{|c|}{ Período estudado } \\
\hline & \multicolumn{2}{|c|}{$1971-1972^{a}$} & \multicolumn{2}{|c|}{$1989-1991^{b}$} \\
\hline & No. & Coeficiente (\%) & No. & Coeficiente (\%) \\
\hline $0-9$ & 242 & - & 299 & - \\
\hline $10-19$ & 209 & 2,9 & 453 & 0,4 \\
\hline $20-29$ & 109 & 22,0 & 372 & 1,6 \\
\hline 30-39 & 98 & 29,6 & 278 & 3,6 \\
\hline $40-49$ & 87 & 49,4 & 222 & 16,7 \\
\hline $50-59$ & 42 & 61,9 & 153 & 42,5 \\
\hline $60+$ & 36 & 25,0 & 174 & 44,8 \\
\hline Total & 823 & 16,6 & 1951 & 10,1 \\
\hline
\end{tabular}

a Soropositividade determinada por reação de fixação de complemento.

b Soropositividade determinada por imunofluorescência indireta.

esta doença na população. A técnica utilizada para identificar chagásicos foi a reação de imunofluorescência indireta para doença de Chagas, de acordo com o método descrito por Camargo (10).

Além dos dados laboratoriais e dos dados obtidos através de exame físico no primeiro estudo, dados foram obtidos em ambos os estudos através de entrevistas feitas pelos autores (por exemplo, dados relativos à caracterização dos indivíduos e dos domicílios) $(6,7)$. Os métodos estatísticos utilizados para a análise dos dados foram os métodos clássicos para descrição de amostras e para teste de associação entre variáveis.

\section{RESULTADOS}

O estudo realizado em 1971-1972, que investigou 823 indivíduos, revelou uma prevalência total de positividade para doença de Chagas de 16,6\%. $\mathrm{O}$ intervalo de confiança (IC) de $95 \%$ foi 14,1 a 19,1 . A taxa de prevalência mais elevada $(61,9 \%)$ ocorreu na faixa etária de 50 a 59 anos. No estudo de 1989-1991, a prevalência total de positividade para doença de Chagas foi de $10,1 \%$ (IC $95 \%=8,8$ a 11,4 ). O qui quadrado $\left(\chi^{2}\right)$ para ambos os estudos foi $\chi_{1 \mathrm{gl}}^{2}=22,41 ; P<0,001$. Em nenhum dos estudos se observou positividade abaixo de 9 anos. Os valores apresentaram uma tendência ascendente à medida em que aumentava a idade, de modo especial na investigação de 1971-1972 (tabela 1 e figura 1). Entre os indivíduos nascidos em Cássia dos Coqueiros (884), o estudo de 19891991 mostrou ausência de positividade nas faixas etárias até 19 anos. Este mesmo estudo registrou um caso de sorologia positiva na faixa entre 20 e 29 anos, correspondendo a um coeficiente igual a $0,5 \%$. Positividade sorológica voltou a se fazer presente apenas após os 38 anos de idade, com valores progressivamente ascendentes até a faixa de 50 a 59 anos de idade (tabela 2 e figura 2).

Embora sem significado estatístico $(P=0,05)$ a prevalência de positividade foi mais elevada entre o sexo masculino em ambos os estudos (figura 3). Para o estudo de 1971-1972, o $\chi^{2}$ de Yates resultou num valor igual a 2,91; para o estudo de 1989-1991, o $\chi^{2}$ mostrou um valor igual a 3,77 $(0,10>P>0,05)$. A comparação das prevalências segundo sexo apenas entre os indivíduos nascidos em Cássia dos Coqueiros não mostrou diferenças significativas $(P>0,10)$. Em ambas as investigações, a positividade se elevou à medida em que aumentava o tempo de residência no Município, com valores sempre mais elevados no estudo de 1971-1972 (tabela 3 e figura 4).

\section{DISCUSSÃO}

A utilização de métodos laboratoriais diferentes nos dois inquéritos em 
FIGURA 1. Coeficientes de prevalência de sorologia positiva para doença de Chagas segundo idade, Cássia dos Coqueiros, São Paulo, Brasil, 1971-1972 e 1989-1991

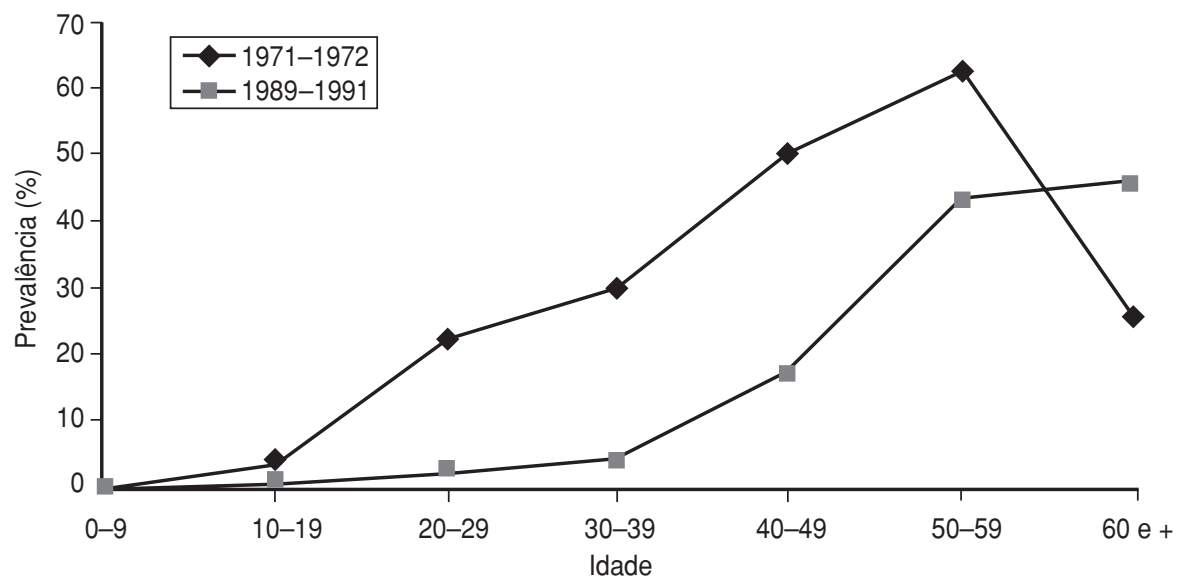

que se baseia este trabalho não compromete a comparabilidade dos resultados, haja visto que uma investigação conduzida em Ribeirão Preto, em 1987, evidenciou concordância acima de $98 \%$ ao comparar resultados dos testes de fixação de complemento e de imunofluorescência indireta para diagnóstico de doença de Chagas, utilizando as mesmas metodologias empregadas nos dois inquéritos aqui discutidos (11). No que diz respeito à doença de Chagas, a região de Cássia dos Coqueiros foi exaustivamente estudada e trabalhada por Pedreira de Freitas (4), a partir do ano de 1945 (3). As primeiras descrições relativas à distribuição da moléstia indicam níveis altíssimos de transmissão, com preva-

TABELA 2. Prevalência de sorologia ${ }^{a}$ positiva para doença de Chagas segundo idade entre os nascidos em Cássia dos Coqueiros, São Paulo, Brasil, 1989-1991

\begin{tabular}{crc}
\hline Idade & No. & Coeficiente (\%) \\
\hline $0-9$ & 55 & - \\
$10-19$ & 210 & - \\
$20-29$ & 195 & 0,5 \\
$30-39$ & 137 & 3,6 \\
$40-49$ & 109 & 19,3 \\
$50-59$ & 80 & 46,3 \\
$60+$ & 98 & 43,9 \\
Total & 884 & 12,1 \\
\hline
\end{tabular}

a Soropositividade determinada por imunofluorescência indireta. lência de resultados sorológicos positivos de 55,5\% numa amostra de 1000 indivíduos testados ao acaso, dentre uma população total de 2700 habitantes (3). Estes estudos iniciais destacam ainda as condições então reinantes favorecedoras da transmissão, representadas pelo elevado número de habitações precárias, grande parte de pau a pique (casas com paredes feitas de varas entrecruzadas, cobertas de barro), onde abundavam exemplares e 1989-1991 de $T$. infestans convivendo em íntimo contato com os seus habitantes $(3,4)$.

As primeiras tentativas de controle de triatomíneos através do uso de inseticidas foram realizadas em Cássia dos Coqueiros já em 1950, muito embora em caráter parcial e visando apenas testar o poder residual de dois produtos: gammexane e rhodiatox (4). Em 1952, o então Serviço Nacional de Malária aplicou $\mathrm{HCH}$ nas paredes internas e dependências de todas as habitações, trabalho que foi repetido em 1954, com o uso de gammexane e DDT pelo então Serviço de Profilaxia da Malária do Estado de São Paulo. A irregularidade destas aplicações fez com que a taxa de infecção entre triatomíneos não fosse sensivelmente alterada, muito embora tenha produzido um impacto considerável em termos de redução do número de domicílios com altos níveis de infestação, quando se comparam observações feitas em 1945 e 1956 (4).

Os trabalhos sistemáticos de aplicação de inseticidas em toda a área foram iniciados em 4 de dezembro de 1956, inicialmente dividindo-se o Município em duas partes similares e aplicando-se dieldrin numa delas e HCH na outra (4). Em virtude dos efei-

FIGURA 2. Coeficientes de prevalência de sorologia positiva para doença de Chagas segundo idade, entre os nascidos em Cássia dos Coqueiros, São Paulo, Brasil, 1971-1972

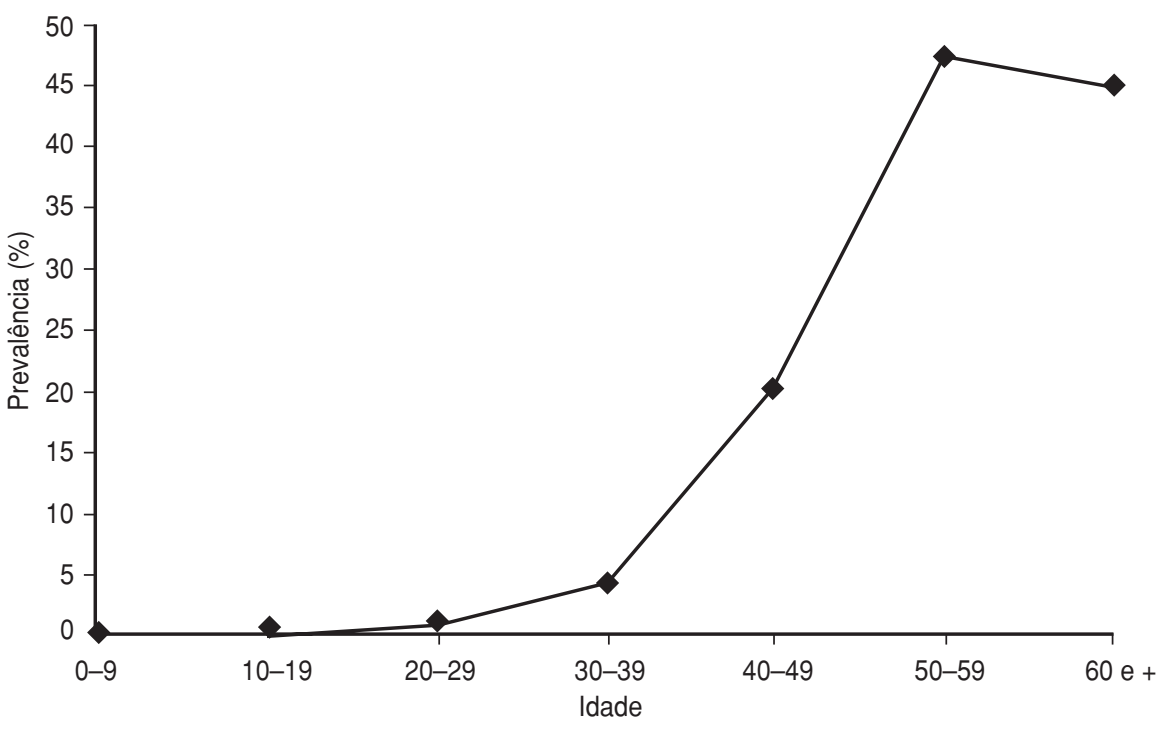


FIGURA 3. Coeficientes de prevalência de sorologia positiva para doença de Chagas segundo sexo, Cássia dos Coqueiros, São Paulo, Brasil, 1971-1972 e 1989-1991

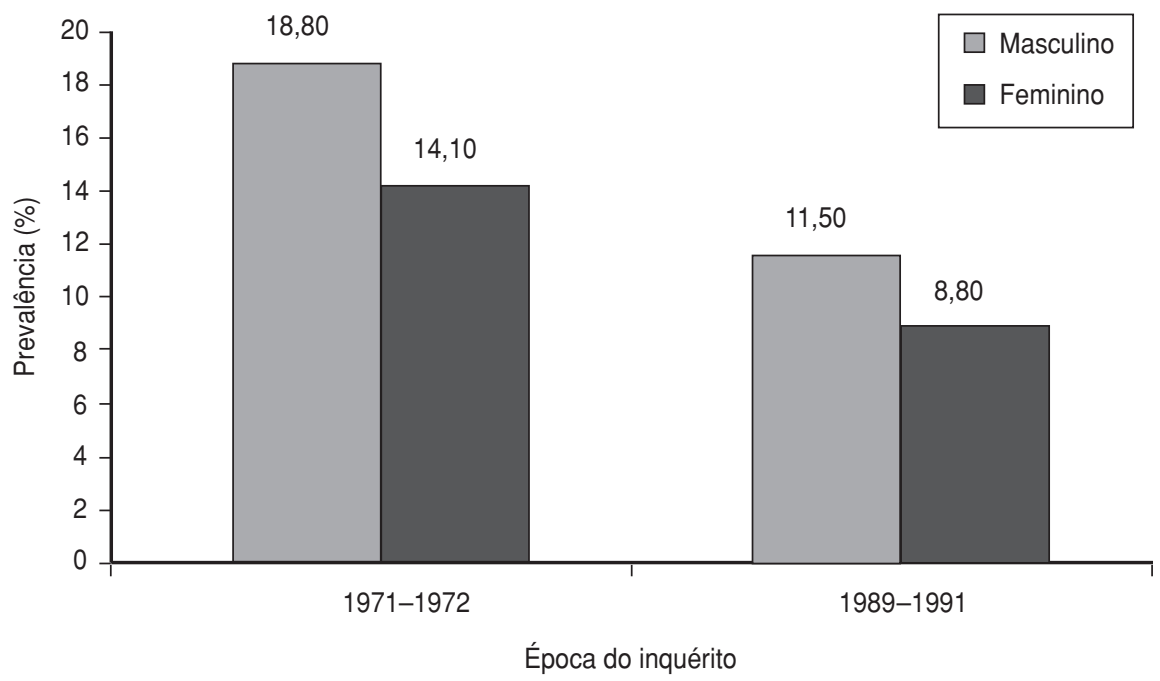

tos indesejáveis decorrentes da aplicação do primeiro produto (basicamente, mortalidade de animais domésticos), optou-se pela suspensão do seu uso e pela adoção do $\mathrm{HCH}$ nos repasses seguintes, em ambas as áreas. Estes repasses, progressivamente espaçados, perduraram até 1961 e resultaram numa redução extremamente rápida dos domicílios positivos para $T$. infestans, o que se verificou já a partir de 1957, logo após as primeiras borrifações (4).

Estas considerações históricas confirmam os resultados descritos no presente trabalho. Com efeito, já no inquérito realizado no início dos anos 70, não se verificou positividade sorológica abaixo de 9 anos de idade. A mesma foi muito baixa entre 10 e 19 anos e subiu consideravelmente nas faixas a partir de 20 anos (tabela 1). No inquérito de 1989-1991, a positividade foi alta apenas para faixas etárias acima de 40 anos (tabela 1), mostrando de maneira inequívoca a redução progressiva do risco de infecção nas coortes formadas por indivíduos mais jovens de ambos os sexos (tabela 1 e figura 1). Efeito similar é observado quando se consideram as prevalências de acordo com o tempo de residência no Município, em ambos os levantamentos (tabela 3 e figura 4). Deve ser ressaltado que esta

TABELA 3. Prevalência de sorologia positiva para doença de Chagas segundo tempo de residência na localidade e o período do estudo, Cássia dos Coqueiros, São Paulo, Brasil

\begin{tabular}{|c|c|c|c|c|}
\hline \multirow[b]{3}{*}{ Idade } & \multicolumn{4}{|c|}{ Período estudado } \\
\hline & \multicolumn{2}{|c|}{$1971-1972^{a}$} & \multicolumn{2}{|c|}{$1989-1991^{b}$} \\
\hline & No. & Coeficiente (\%) & No. & Coeficiente (\%) \\
\hline $0-14$ & 467 & 4,7 & 858 & 3,4 \\
\hline $15-24$ & 149 & 12,7 & 417 & 5,3 \\
\hline 25-39 & 108 & 44,4 & 336 & 6,3 \\
\hline $40+$ & 99 & 48,4 & 126 & 37,1 \\
\hline Total & 823 & 16,6 & 1951 & 10,1 \\
\hline
\end{tabular}

\footnotetext{
a Soropositividade determinada por reação de fixação de complemento.
}

${ }^{\mathrm{b}}$ Soropositividade determinada por imunofluorescência indireta.

análise engloba os habitantes do Município como um todo, sem discriminar o local de nascimento.

Entre pessoas nascidas em Cássia dos Coqueiros (tabela 2 e figura 2), o estudo constatou positividade apenas a partir dos 38 anos de idade. A única exceção foi um jovem de 24 anos, aparentemente saudável, que sofreu uma súbita parada cardíaca irreversível em 1989. O conhecimento da sua positividade para moléstia de Chagas se deu apenas em 1993, quando uma amostra do seu sangue, mantido estocado, foi submetida ao exame específico. Este indivíduo nunca havia recebido transfusão sangüínea nem saído de Cássia dos Coqueiros, residindo em habitação rural com boas condições gerais, sem qualquer sinal de presença de triatomíneos. A análise sorológica revelou-se negativa em oito membros da mesma família, que sempre habitaram a mesma casa. Além disso, a negatividade de todos os outros 591 indivíduos abaixo de 38 anos participantes do estudo justifica que se pense ser este um caso excepcional, cuja infecção provavelmente ocorreu fora do domicílio.

À luz dos resultados desta investigação, com base nas faixas etárias que apresentaram positividade e na época da coleta do material, pode-se considerar que a transmissão de doença de Chagas através do contato de triatomíneos com seres humanos no ambiente domiciliar, em Cássia dos Coqueiros, possivelmente deixou de ocorrer em algum momento entre 1952 e final de 1954, período que corresponde justamente à época em que foram realizadas as duas primeiras borrifações cobrindo a totalidade da área de estudo (4). Este achado é surpreendente, uma vez que as primeiras borrifações foram espaçadas entre si por um período de 2 anos e não foram capazes de eliminar a presença do $T$. infestans do ambiente domiciliar. Com efeito, a presença do T. infestans foi referida por Pedreira de Freitas quando dos trabalhos que antecederam a sistematização dos repasses, a partir do final de 1956. Entretanto, o autor faz a ressalva de que, nesta época, contrastando nitidamente com o observado quando iniciou os trabalhos na área, em 1945, 
FIGURA 4. Coeficientes de prevalência de sorologia positiva para doença de Chagas segundo tempo de residência na localidade Cássia dos Coqueiros, São Paulo, Brasil, 1971-1972 e 1989-1991

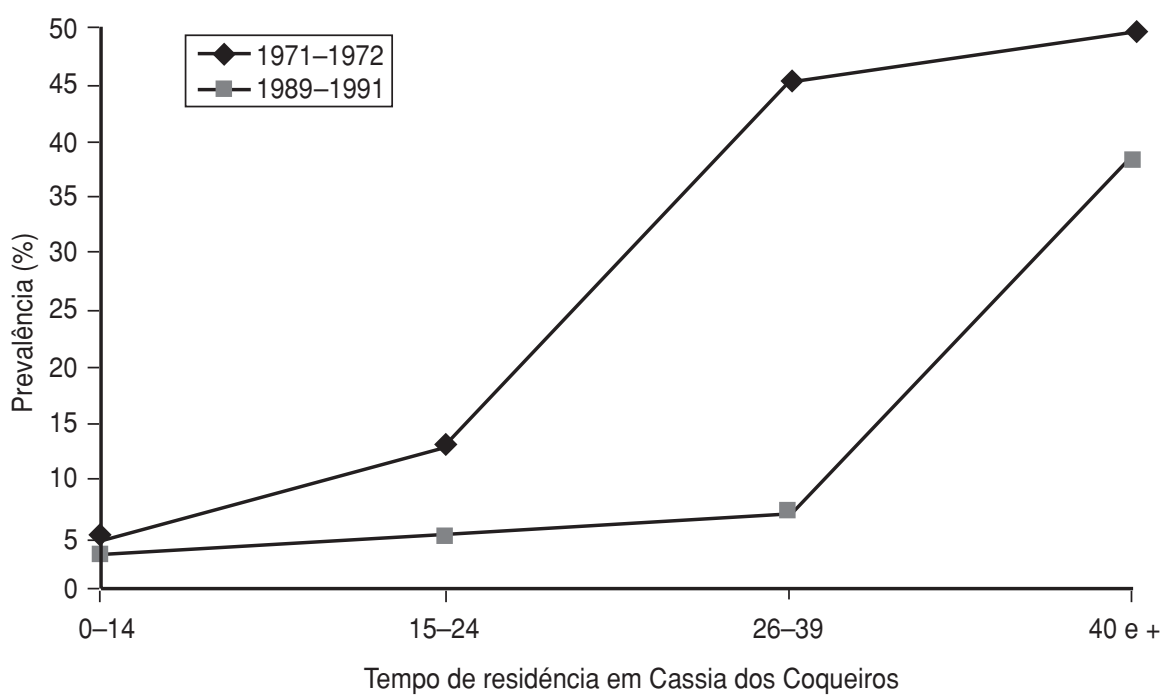

foram raras as casas altamente infestadas por triatomíneos. Esta redução dos níveis de infestação domiciliar foi por ele atribuída "ao emprego de inseticidas potentes, ainda que de forma irregular, como vinha sucedendo em Cássia dos Coqueiros" (4).

Se os últimos casos autóctones de fato ocorreram antes do final de 1954, isto significaria que a simples redução do número de domicílios altamente infestados teria sido capaz de interromper a transmissão da doença de Chagas em Cássia dos Coqueiros. Esta conclusão contraria o conhecimento oficial, que localiza o fim da transmissão endêmica da doença em meados dos anos 60, como decorrência da apli- cação sistematizada de inseticidas associada à melhoria das habitações e ao êxodo rural. Causa surpresa que isto possa ter ocorrido já em 1954, numa situação em que a dispersão de T. infestans ainda era bastante evidente. Igualmente surpreendente seria o fato de que a transmissão teria deixado de ocorrer num curto espaço de tempo, sem que as condições sócioeconômicas da população tivessem experimentado melhoria significativa, e antes da ocorrência de um efetivo esvaziamento das áreas rurais. Este achado sugere que a aplicação de inseticidas de ação residual é eficaz para a interrupção da transmissão da doença de Chagas, ainda que essa aplicação se faça de maneira não rigorosamente sistematizada e mesmo na ausência de fatores de natureza social e econômica que dificultem o contato do ser humano com o vetor. Embora os dados do presente trabalho demonstrem a excelente resposta a uma medida de intervenção puramente biológica, é inegável que a interrupção da transmissão só pôde ser consolidada de maneira definitiva pelas alterações sócio-econômicas e demográficas que se seguiram e impuseram uma barreira eficaz à manutenção da endemia chagásica na área estudada (5).

\section{REFERÊNCIAS}

1. Brasil, Ministério da Saúde, Superintendência de Campanhas de Saúde Pública (SUCAM). Doença de Chagas — textos de apoio. Brasília: SUCAM; 1989.

2. Brasil, Ministério da Saúde, Superintendência de Campanhas de Saúde Pública (SUCAM). Doença de Chagas - clínica e terapêutica. Brasília: SUCAM; 1990.

3. Pedreira de Freitas JL. Aspectos da epidemiologia da moléstia de Chagas no Estado de São Paulo. Rev Clinica Sao Paulo 1950;26 (11/12):181-190.

4. Pedreira de Freitas JL. Importância do expurgo seletivo dos domicílios e anexos para a profilaxia da moléstia de Chagas pelo combate aos triatomíneos [tese de livre docência]. Ribeirão Preto: Faculdade de Medicina de Ribeirão Preto, Universidade de São Paulo; 1963.

5. Silva LJ. Evolução da doença de Chagas no Estado de São Paulo [tese de doutorado]. Ribei- rão Preto: Faculdade de Medicina de Ribeirão Preto, Universidade de São Paulo; 1981.

6. Nogueira JL. Levantamento epidemiológico sobre cardiopatias e pressão arterial na população do Município de Cássia dos Coqueiros, SP, Brasil [tese de doutorado]. Ribeirão Preto: Faculdade de Medicina de Ribeirão Preto, Universidade de São Paulo; 1972.

7. Passos ADC. Estudo epidemiológico sobre a hepatite B em Cássia dos Coqueiros, SP, Brasil [tese de doutorado]. Ribeirão Preto: Faculdade de Medicina de Ribeirão Preto, Universidade de São Paulo; 1991.

8. Pedreira de Freitas JL. Reação de fixação de complemento para diagnóstico da moléstia de Chagas pela técnica quantitativa [tese de doutorado]. São Paulo: Faculdade de Medicina da Universidade de São Paulo; 1951.

9. Almeida JO, Freitas JLP, Brandão H. Complement fixation test with a triple antigen for syphilis, tuberculosis, leprosy or Chagas' disease in blood banks. Am J Trop Med Hyg 1954;3:490-494.

10. Camargo ME. Fluorescent antibody test for the serodiagnosis of American Trypanosomiasis: Technical modification employing preserved culture forms of Trypanosoma cruzi in a slide test. Rev Inst Med Trop Sao Paulo 1966;8:227-234.

11. Figueiredo JFC. Estudo do emprego simultâneo das reações de fixação de complemento e imunofluorescência indireta na exclusão de doadores de sangue chagásicos [tese de doutorado]. Ribeirão Preto: Faculdade de Medicina de Ribeirão Preto, Universidade de São Paulo; 1987.

Manuscrito recebido em 31 de janeiro de 1996. Aceito para publicação em 31 de maio de 1996. 
ABSTRACT In order to assess the evolution of seropositivity for Chagas' disease in the rural community of Cássia dos Coqueiros, in São Paulo state, we compared two cross-sectional studies performed at that site in 1971-1972 and in 1989-1991. In the first survey the complement fixation test was used to determine seropositivity for the disease. In this study, the total prevalence of Chagas' disease was found to be $16.6 \%$, with values ranging from 2.9 to $61.9 \%$ (in the 10-19 and 50-59 year age groups, respectively). In the 1989-1991 study, in which indirect immunofluorescence was used, the total prevalence dropped to $10,1 \%$, with a minimum of $0.4 \%$ (in the $10-19$ year age group) and a maximum of $44.8 \%$ (in persons over 60 years of age). Among subjects born in Cássia dos Coqueiros, the 1989-1991 study showed seropositivity after age 38, with only one exception (a 24-year-old man). The drop in the seropositivity index for Chagas' disease in Cásia dos Coqueiros during the period under study can be partially attributed to socioeconomic factors, such as improved living conditions and the progressive abandonment of rural areas. Thus, our results point to the spraying of households with residual insecticides, which began in 1950, as the pivotal factor in the fight against triatomine bugs and in disease control. Theses results suggest that transmission may have been interrupted as early as the latter part of 1954 and later consolidated as a result of improved socioeconomic conditions and the elimination of Triatoma infestans from the study area.

\section{Evolution of seropositivity for community in Brazil}

\title{
Cuarta Conferencia Internacional sobre Fiebre Hemorrágica con Síndrome Renal y Hantavirus
}

\author{
Fechas: $\quad 5$ a 7 de marzo de 1998 \\ Lugar: $\quad$ Atlanta, Georgia, Estados Unidos de América \\ Tema: $\quad$ Síndromes renales en casos de fiebre hemorrágica \\ e infecciones por hantavirus
}

Esta Conferencia, que ha sido organizada con el apoyo de los Centros para el Control y la Prevención de Enfermedades en Atlanta, Georgia, Estados Unidos y que precederá a la Conferencia Internacional sobre Enfermedades Infecciosas Emergentes en la misma ciudad, tiene por objetivo facilitar el intercambio de información científica en las siguientes áreas: 1) aspectos clínicos; 2) diagnóstico de laboratorio; 3) patogenia y respuesta inmunitaria; 4) ecología de Ios hantavirus; 5) epidemiología de los hantavirus; 6) biología molecular e interacciones celulares; 7) educación y prevención en salud; y 8) desarrollo de fármacos y vacunas antivirales. La reunión estará compuesta de sesiones plenarias a las que asistirán invitados especiales y en las que se presentarán comunicaciones cuyos resúmenes hayan sido aceptados.

\author{
Información: \\ Amy L. Corneli \\ Special Pathogens Branch \\ Centers for Disease Control and Prevention \\ 1600 Clifton Road, M/S A26 \\ Atlanta, Georgia 30333, USA \\ Teléfono: (404) 6391510 \\ Fax: (404) 6391509
}

